Recibido: 10 de setiembre del 2016

Aprobado: 26 de setiembre del 2016

\title{
ModelO DE REFERENCIA DE GOBIERNO DE LAS TECNOLOGÍAS DE LA INFORMACIÓN PARA INSTITUCIONES UNIVERSITARIAS ${ }^{1}$
}

\author{
Manuel Yrigoyen Quintanilla \\ Myrigoye@correo.ulima.edu.pe \\ Escuela de Ingeniería de la Universidad de Lima. Lima, Perú
}

\section{Resumen}

El Gobierno de las Tecnologías de la Información (TI) se desarrolló con el objetivo de ayudar a que las organizaciones hagan uso efectivo y eficiente de ellas, apoyándolas en el cumplimiento de sus objetivos estratégicos, con la participación y compromiso pleno de la alta dirección. La premisa de la investigación es que este enfoque puede y debe ser aplicado en las instituciones universitarias, para lo cual se realizó un análisis del estado del arte, y de los principales marcos de referencia y estándares aplicables disponibles en el mercado. A partir del análisis realizado, se propone un Modelo de Gobierno de TI (GTIUP) aplicable a la universidad peruana, así como dos herramientas de apoyo para su implementación.

Palabras clave: gobierno empresarial / gobierno corporativo / gobierno de las TI / gestión de las TI I marcos de referencia de TI / estándares de TI

\section{Abstract}

Government in information techonologies-IT was developed to help organizations make better and more efficient use of IT, supporting them to achieve their strategic objectives with the participation and full commitment of senior management. The premise of this investigation is that this approach can and should be applied in university institutions; accordingly, an analysis was carried out about the state of the art of this concept and the main reference frameworks and standards to be applied available on the market. As a result of this analysis, an IT Governance model (GTIUP) is proposed, applicable to Peruvian universities as well as two supporting tools for its implementation.

Keywords: business governance / corporate governance / IT governance / IT management / IT frameworks / IT standards

1 El presente artículo se basa en la investigación realizada por el suscrito en el año 2015, auspiciado por el Instituto de Investigación Científica de la Universidad de Lima (IDIC). 


\section{Gobierno empresarial}

El gobierno de las tecnologías de la información constituye una parte integral de la empresa; es el sistema por el cual las TI de una organización son dirigidas y controladas por sus niveles más altos, buscando un alineamiento entre los objetivos de negocio y los de las TI. Debido a la fuerte dependencia de los negocios a las TI, los aspectos de gobierno empresarial no pueden ser resueltos sin considerarlas. El gobierno empresarial es el que orienta y controla el gobierno de las TI. ISACA lo define de la siguiente manera:

Gobierno Empresarial es el conjunto de prácticas y responsabilidades ejercidas por la dirección de la empresa y la gestión ejecutiva con la meta de proporcionar dirección estratégica, asegurando que los objetivos sean logrados, garantizando que los riesgos sean gestionados apropiadamente y verificando que los recursos de la empresa sean usados responsablemente (ISACA, 2014a, p.7).

El término "gobierno" es definido como sigue:

Gobierno deriva del verbo griego kubernáo que significa dirigir. Un sistema de gobierno permite a las múltiples partes interesadas en una empresa tener una voz organizada en la evaluación de las condiciones y opciones, estableciendo la dirección y supervisando el rendimiento respecto a los objetivos de la empresa. Establecer y mantener el enfoque de gobierno adecuado es responsabilidad del consejo de administración u órgano equivalente. El gobierno asegura que las necesidades, condiciones y opciones de las partes interesadas son evaluadas para determinar los objetivos acordados y equilibrados de empresa que han de ser alcanzados; establecer la dirección mediante la priorización y toma de decisiones; y supervisando el rendimiento y el cumplimiento respecto a la dirección y objetivos acordados (ISACA, 2012a, p. 13).

La International Federation of Accounts (IFAC) publicó un reporte de una encuesta que se realizó para explorar el concepto de gobierno empresarial. El reporte menciona lo siguiente:

Se identificaron dos dimensiones de gobierno empresarial que son la conformidad y el desempeño, indicando que estas dos dimensiones deben estar en balance. La Conformidad es también llamada Gobierno Corporativo, y cubre aspectos como la estructura directiva, roles y remuneraciones ejecutivas. La dimensión de Desempeño está enfocada en la estrategia y la creación de valor, y en ayudar a la dirección en la toma de decisiones estratégicas, entender su apetito de riesgo y los impulsores claves de desempeño. El Gobierno Empresarial con foco en los aspectos de Conformidad y el Desempeño del negocio, asegura que la empresa no pierda de vista su perspectiva. Otros reportes y marcos de referencia usan una terminología diferente y se refieren con el término de Gobierno Corporativo a lo que la IFAC denomina Gobierno Empresarial (ISACA, 2014a). 


\section{Gobierno de las tecnologías de la información}

Según ISACA (2012b), el concepto de "gobierno" se define de la siguiente manera:

El gobierno asegura que se evalúan las necesidades, condiciones y opciones de las partes interesadas para determinar que se alcanzan las metas corporativas equilibradas y acordadas; estableciendo la dirección a través de la priorización y la toma de decisiones; y midiendo el rendimiento y el cumplimiento respecto a la dirección y metas acordadas. En la mayoría de las empresas, el gobierno es responsabilidad del consejo de administración bajo la dirección de su presidente (p. 31).

Asimismo, ISACA (2012a), define así el gobierno de las TI:

El gobierno de las TI no es una disciplina aislada, sino una parte integral del gobierno empresarial. Mientras que la necesidad para el gobierno a nivel de empresa se debe principalmente a la entrega de valor para las partes interesadas y la demanda de transparencia y gestión eficaz del riesgo de la empresa, las oportunidades, los costes y los riesgos importantes asociados; en el caso del gobierno de las TI se requiere de un enfoque dedicado, a la vez que integrado. El gobierno de las TI permite a la empresa aprovechar al máximo las ventajas de las TI, maximizar los beneficios, capitalizando las oportunidades y ganando ventaja competitiva (p. 13).

Entonces, se puede decir, el gobierno de las TI está relacionado con la entrega de valor de las $\mathrm{TI}$ al negocio, esto es, la realización de beneficios optimizando el riesgo y haciendo un uso eficiente y efectivo de los recursos de la organización, de modo tal que se logre el alineamiento entre las TI y empresa.

\section{Gestión de las tecnologías de la información}

Conforme se manifiesta en ISACA (2012b), existe una clara distinción entre gobierno y gestión, indicando que se trata de dos disciplinas diferentes, con actividades y estructuras diferentes, que sirven para diferentes objetivos. Además, menciona que: "la gestión planifica, construye, ejecuta y controla actividades alineadas con la dirección establecida por el cuerpo de gobierno para alcanzar las metas empresariales" (p. 31).

Se puede concluir entonces que la gestión de las TI se refiere al uso efectivo y eficiente de las TI para crear soluciones de negocio que den soporte a los objetivos de la empresa, dicha gestión estará sujeta a la dirección y control por parte del órgano de gobierno de las TI de la organización, que recae en el cuerpo de dirección de la empresa. 


\section{COBIT 5: Marco de referencia de gobierno y gestión de las TI}

Existen muchos marcos de referencia de Gobierno de las TI, siendo el principal el Control Objetives for Information Technology (COBIT), de ISACA, que lanzó al mercado en abril de 2012 su última versión. COBIT 5 es un marco de referencia libre, que describe las mejores prácticas de gobierno y gestión de las TI, organizado en una estructura conceptual basada en procesos. COBIT 5 brinda un marco de trabajo general que puede ser utilizado por cualquier tipo de empresa para implementar gobierno y gestión de las TI como parte del proceso de implantación de gobierno empresarial. Este ayuda a las empresas a crear valor mediante el uso de las TI, manteniendo un balance entre los beneficios, riesgos y recursos utilizados. Además, identifica treinta y siete procesos de TI organizados en cinco dominios: uno para el tema de gobierno y el resto para los temas de gestión. La figura 1 muestra el modelo de referencia de procesos.

Figura 1. Modelo de referencia de procesos de COBIT 5

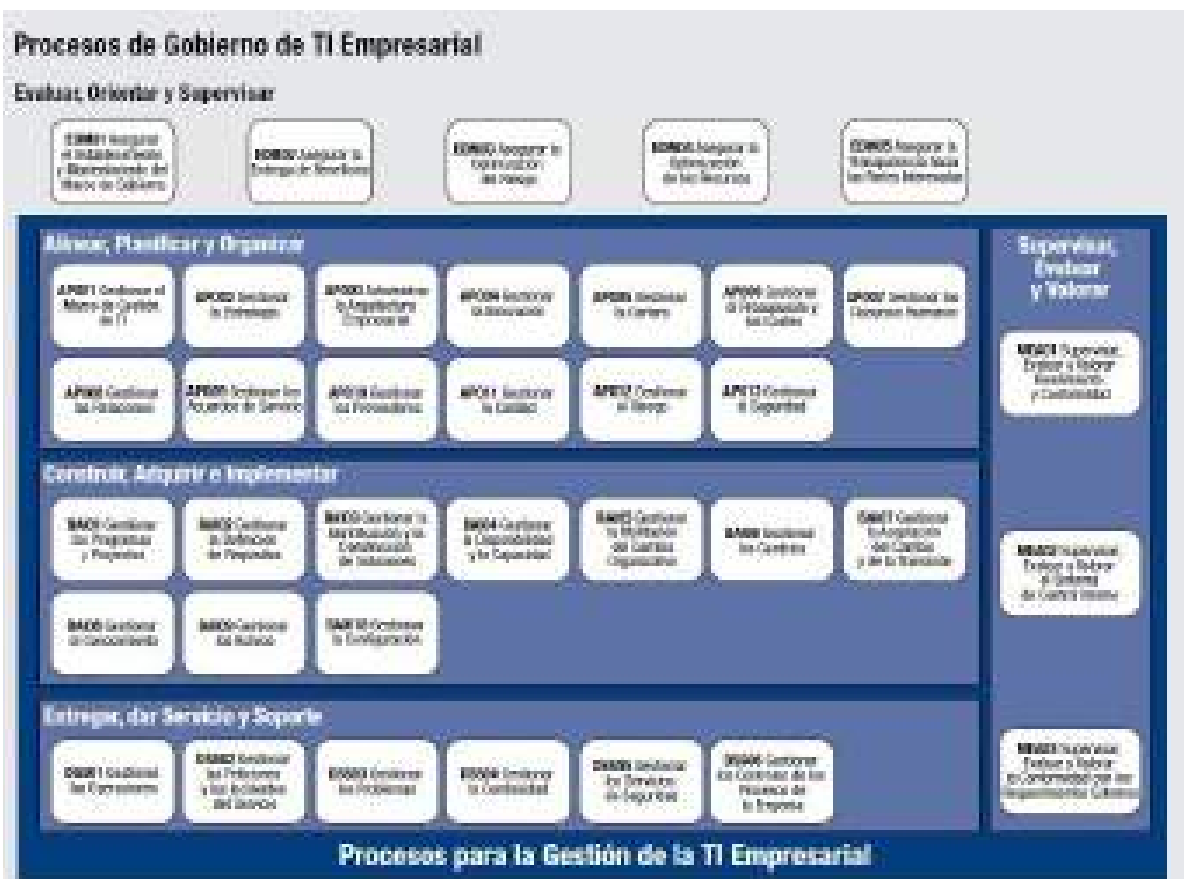

Fuente: ISACA (2012b). Un marco de negocio para el gobierno y la gestión de la empresa.

COBIT 5 establece que las TI deben ser gobernadas y gestionadas de forma holística para toda la empresa, considerando tanto las áreas funcionales de negocio como las de TI, teniendo en cuenta los intereses de los involucrados (stakeholders) internos y externos. Para lograrlo utiliza lo que se denomina "habilitador" (enabler), que es definido como los facto- 
res que individual o colectivamente influyen en que funcione; en este caso el gobierno y la gestión de las TI en la empresa. En la figura 2 se pueden ver estos habilitadores.

Figura 2. Habilitadores de COBIT 5

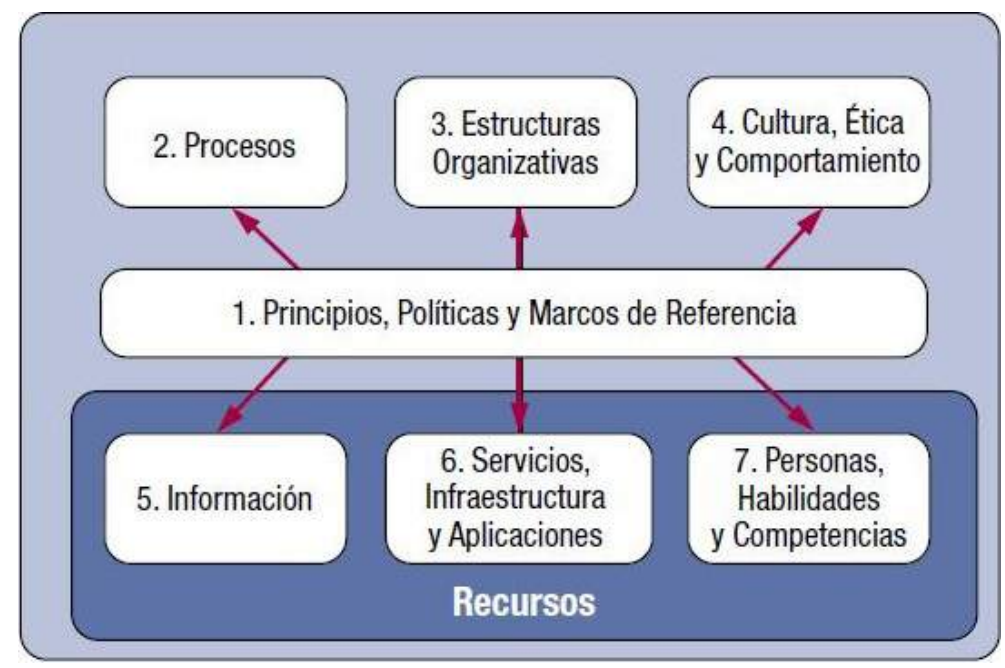

Fuente: ISACA (2012b). Un marco de negocio para el gobierno y la gestión de la empresa

Los componentes 2, 3 y 4 son los que la ciencia de la administración (management) denomina "sistema organizacional".

\section{ISO/IEC 38500: Norma de gobierno corporativo de las TI}

A continuación se presenta un resumen de la norma en base a la información de ISO/IEC 38500, elaborado por la International Organization for Standardization/ International Electrotechnical Commission (ISO/IEC) (2008):

La norma proporciona los principios para que el cuerpo de gobierno efectúe un uso eficaz y eficiente de las TI en sus organizaciones. Esta norma es aplicable a todo tipo de organizaciones sean públicas o privadas de todos los tamaños, entidades gubernamentales y entidades sin ánimo de lucro; siendo aplicable con independencia del grado de utilización de las TI. Un adecuado gobierno corporativo de las TI ayuda a garantizar que su uso contribuya positivamente al desempeño de la organización, para lo cual es necesario lo siguiente:

- Una implementación y explotación adecuada de los activos de las TI.

- Claridad de responsabilidad e imputabilidad tanto para el uso como para la provisión de las TI destinados al logro de los objetivos empresariales.

- Continuidad y sostenibilidad del negocio. 
- La alineación de las TI con las necesidades del negocio.

- La asignación eficiente de recursos.

- La innovación en los servicios, mercados y negocios.

- Las buenas prácticas en las relaciones con las partes interesadas.

- La reducción de los costes de una organización.

- La consecución real de los beneficios aprobados para cada inversión en TI.

La norma establece seis principios de gobierno corporativo de las TI. Estos expresan el comportamiento deseable para orientar la toma de decisiones. Las definiciones de cada principio se refieren a lo que debería suceder, pero no prescribe como, cuando o quién los pondría en práctica, ya que estos aspectos dependen de la naturaleza de la organización que los implanta. Estos principios son:

- $\quad$ Principio 1: Responsabilidad

- $\quad$ Principio 2: Estrategia

- Principio 3: Adquisición

- Principio 4: Desempeño

- Principio 5: Cumplimiento

- Principio 6: Conducta humana

Como se señala en ISO/IEC (2008, pp. 1-8), el modelo de referencia está compuesto de tres áreas principales que se encargan de las tareas que se indican a continuación:

- Evaluar el uso actual y futuro de las TI.

- Dirigir la preparación y ejecución de planes y políticas para asegurar que el uso de las TI satisface los objetivos de la organización.

- Monitorizar el cumplimiento de las políticas y el desempeño según lo planificado.

En la figura 3 se muestra el modelo de gobierno de las TI. 
Figura 3. Modelo de gobierno corporativo de las TI

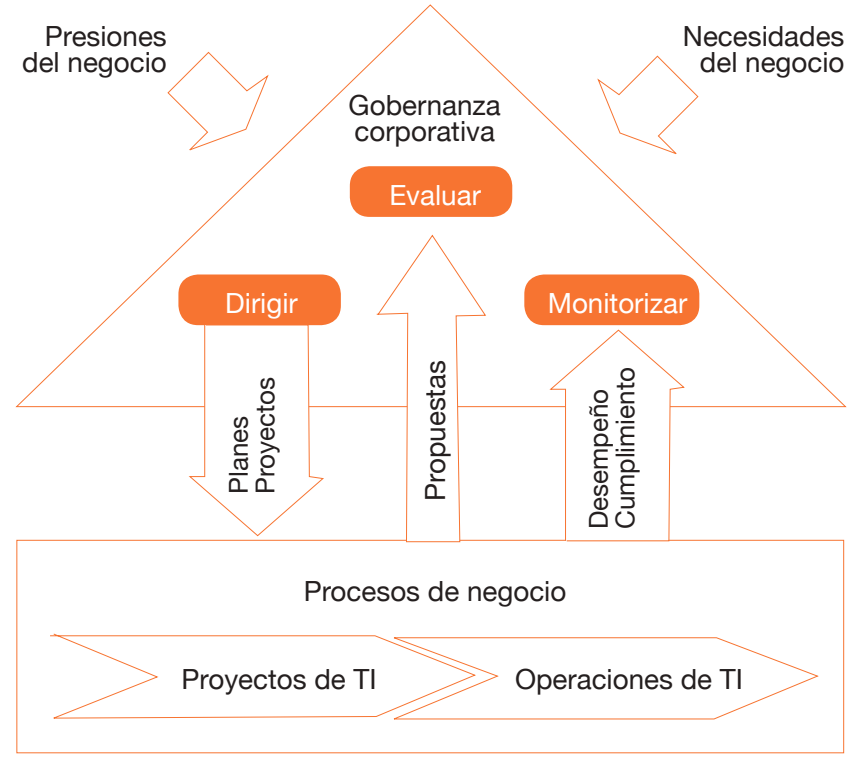

Fuente: ISO/IEC (2008)

\section{Modelo de referencia propuesto}

Dado que el desarrollo e implantación del enfoque de gobierno de las TI es muy incipiente en los diferentes sectores de país, y más aún en el sector de la educación universitaria, se toma como base información sobre la situación global de este enfoque a nivel mundial; de acuerdo a los estudios efectuados por el Instituto de Gobierno de TI e ISACA conjuntamente con organizaciones o universidades asociadas, referentes reconocidos en la materia que realizan estudios periódicos para tomar el pulso respecto al desarrollo e implantación del gobierno de las TI en las organizaciones. A continuación se presentan los estudios analizados:

- Encuesta para determinar los beneficios de la implantación de gobierno de las TI en las organizaciones. Llevada a cabo por ISACA el año 2014, conforme se muestra en el documento ISACA (2014b).

- Investigación de mercado sobre gobierno de las TI, cuarta edición. Llevada a cabo por la compañía PricewaterhouseCoopers de Bélgica el año 2010, por encargo del Instituto de Gobierno de TI, conforme se muestra en el documento IT Governance Institute (2011).

- Estudio de evaluación comparativa y de valor de negocio de COBIT 5, efectuada por la Escuela de Negocios de la Universidad de Antwerp de Bélgica por encargo de ISACA el año 2015, conforme se muestra en el documento ISACA (2015). 
En base al análisis de los estudios citados, se señala:

i El enfoque de Gobierno de las TI es beneficioso para las instituciones universitarias y su implementación ayudará a lograr la integración entre las TI y la universidad, a mejorar la gestión de los riesgos de las TI y a propiciar la participación de los niveles más altos de la institución educativa en la dirección y control de las TI de la universidad.

ii. Las TI son muy valiosas para la estrategia y visión de la empresa en general y de las instituciones universitarias en particular. Su contribución a la organización es ampliamente reconocida porque son las impulsoras más importantes de la implementación de gobierno de las TI, del alineamiento de las TI con el negocio y de la gestión de costos.

iii. Los marcos de referencia, normas o estándares de gobierno de las TI (como COBIT 5 y la ISO/IEC 38500, entre otras) son los habilitadores más destacados para un efectivo gobierno de las TI. Las políticas y estándares de las TI son las prácticas más comunes de gobierno de las TI, seguido de la definición y gestión de procesos de TI. Los principales desafíos que se experimentan en la implementación de mecanismos de gobierno de las TI son la comunicación y la gestión del cambio.

iv. Los siete habilitadores de COBIT 5 son de mucha utilidad, al igual que su enfoque holístico e interrelacionado. Sin embargo, los habilitadores más relevantes son: información, personas, habilidades, competencias y procesos. Se sugiere que los niveles de implementación de los procesos de gobierno sean percibidos como inferiores a la de los procesos de Gestión. Los procesos con mayor nivel de implementación en las organizaciones son: EDM05 (asegurar la transparencia de los involucrados), APO06 (gestionar el presupuesto y los costos), AP013 (gestionar la seguridad), BAl01 (gestión de programas y proyectos), BAl09 (gestión de activos), DSS02 (gestión de requerimientos de servicio e incidentes), DSS01 (gestionar las operaciones) y MEA03 (monitorizar, evaluar y valorar el cumplimiento de los requerimientos externos).

Según ISACA (2012b, p.7):

Las empresas existen para crear valor para sus accionistas. En consecuencia, cualquier empresa, comercial o no, tendrá a la creación de valor como un objetivo de Gobierno. Creación de valor significa conseguir beneficios a un coste óptimo de los recursos mientras se optimiza el riesgo. Los beneficios pueden tomar muchas formas, por ejemplo, financieros para las empresas comerciales o de servicio público para entidades gubernamentales. 
La norma ISO/IEC 38500 (2008), establece que "un adecuado gobierno corporativo de las Tl ayuda a garantizar que el uso de las TI contribuya positivamente al desempeño de la organización". Esta contribución se manifiesta mediante:

- Una implementación y explotación adecuada de los activos de las TI.

- Claridad de responsabilidad e imputabilidad tanto para el uso como para la provisión de TI para el logro de los objetivos empresariales.

- Continuidad y sostenibilidad del negocio.

- La alineación de las TI con las necesidades del negocio.

- La asignación eficiente de recursos.

- La innovación en los servicios, mercados y negocios.

- Las buenas prácticas en las relaciones con las partes interesadas.

- La reducción de los costes de una organización.

- La consecución real de los beneficios aprobados para cada inversión en TI.

Conforme a ISACA (2012a, p.13), "el gobierno de TI permite a la empresa aprovechar al máximo las ventajas de las TI, maximizar los beneficios, capitalizando las oportunidades y ganando ventaja competitiva".

En base al análisis efectuado, se infiere que la implementación de un sistema de gobierno de las $\mathrm{Tl}$ en la universidad peruana le debe proporcionar valor, mediante una adecuada utilización de TI que contribuya a la obtención de beneficios, optimizando los riesgos y el uso de los recursos. No obstante, el uso de las TI en las universidades peruanas está muy diferenciado en relación directa a la diversidad, tamaño y complejidad de las mismas. Hay pocas universidades, que generalmente pertenecen al sector privado, que tienen implementados sistemas de información con una buena infraestructura y aplicaciones de soporte para sus labores académicas, administrativas y de investigación; sin embargo la mayoría no cuenta con la infraestructura ni los sistemas adecuados. En este contexto es que el concepto y enfoque de gobierno de las TI no es generalmente conocido ni entendido, salvo tal vez pocas excepciones. En este sentido, el objetivo de la investigación es plantear un modelo de gobierno de las TI aplicable a la universidad peruana con las siguientes características:

- Que se convierta en el instrumento a través del cual se entienda la importancia del gobierno de las TI para la universidad. 
- Que fomente la participación del cuerpo directivo y del personal encargado de gestionar las TI en las universidades.

- Que proporcione un esquema conceptual y una guía básica de implementación.

- Que cuente con herramientas básicas que permitan iniciar el proceso.

- Que sirva para la difusión del enfoque de gobierno de las TI y del marco de referencia COBIT 5 en el sector universitario.

El modelo de gobierno de las TI para las universidades peruanas estará basado en el marco de referencia COBIT 5, el cual está alineado al estándar ISO/IEC 38500. Si bien es cierto que algunas organizaciones no utilizan este marco por considerarlo demasiado complejo, lo cierto es que puede y debe ser adaptado a las necesidades, al entorno de cada realidad. En la propuesta se utiliza COBIT 5 con una adaptación básica inicial, la que podrá ir ampliándose para aprovechar más características del marco de referencia en el tiempo. El modelo está compuesto de tres etapas, las que se describen a continuación:

- La primera etapa está enfocada en la evaluación y mejora de los cinco procesos de gobierno de las TI definidos en el dominio, con el objetivo de que los procesos logren el nivel de capacidad uno (proceso ejecutado), vale decir que los procesos logren sus metas.

- La segunda etapa se centra en la identificación, evaluación y mejora de los procesos de TI relevantes de los cuatro dominios de gestión. Para identificar estos procesos se utilizará la cascada de metas de COBIT 5. Al igual que en la etapa primera del modelo, el objetivo es que los procesos identificados deben lograr el nivel de capacidad uno. Dada la cantidad de procesos involucrados (treinta y dos procesos en los cuatro dominios), las actividades de identificación y mejora se desarrollarán en distintas fases, considerando en cada fase no más de cinco procesos y en un periodo que no supere los seis meses.

- La tercera etapa consiste en la mejora continua del nivel de capacidad de los procesos de gobierno y gestión, cuyo objetivo es mejorar su nivel de capacidad por encima del nivel uno, en función a las necesidades de la universidad. Como en la segunda etapa, las actividades de mejora se llevarán a cabo en distintas fases. Esta etapa puede implementarse en forma paralela a la segunda etapa.

El modelo propuesto comienza las actividades de mejora de procesos con los cinco procesos de gobierno, pues un factor clave para el éxito del proceso de implementación de gobierno de las TI es la involucración de los niveles más altos de la organización, situación 
que se logrará al implementar los procesos de gobierno. Con estos procesos implementados en el nivel de capacidad uno (proceso ejecutado) se contará con la base necesaria para que los niveles más altos de la institución puedan evaluar, dirigir y controlar las actividades de las TI, garantizando el alineamiento con los objetivos de la institución. En la figura 4 se presenta un esquema del modelo.

Figura 4. Modelo de gobierno de las TI (GTIUP)

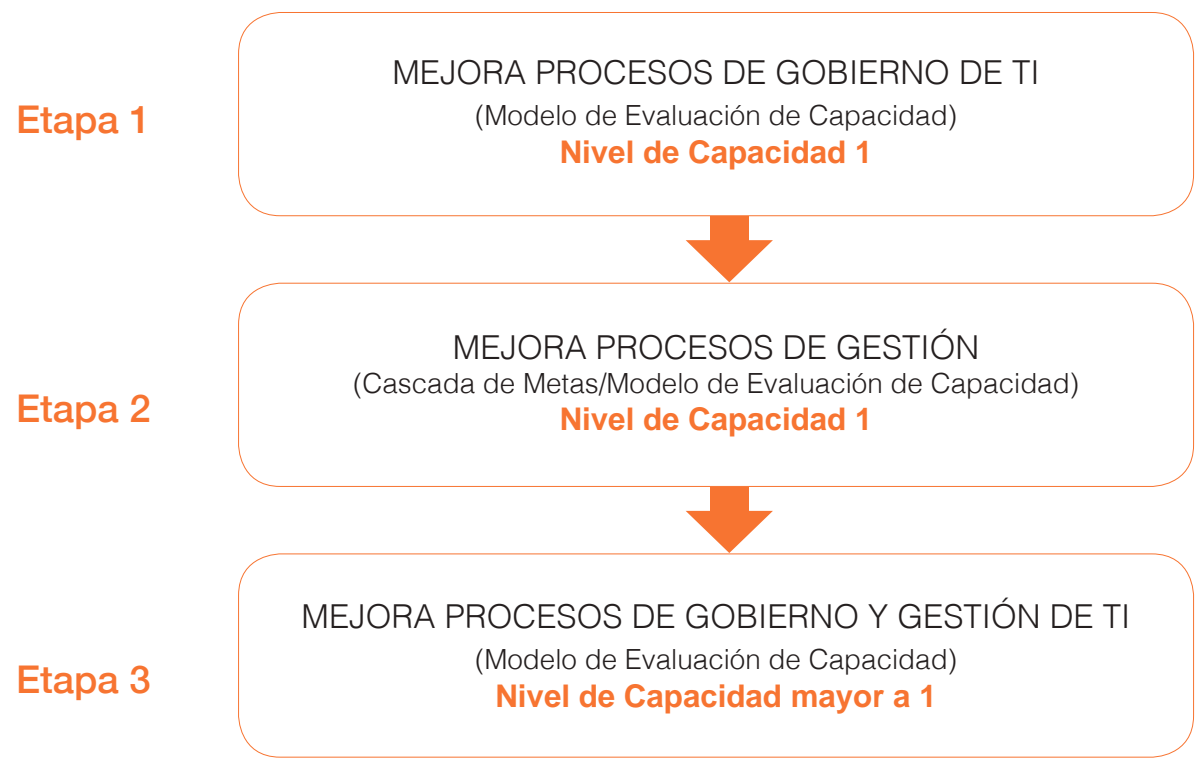

Elaboración propia

\subsection{Modelo de gobierno de las TI (GTIUP). Etapa 1 \\ El Modelo está compuesto de los siguientes tres niveles:}

\section{Nivel 1}

Está integrado por los procesos de gobierno de COBIT 5. Estos cinco procesos deben ser analizados e implementados en el nivel de capacidad uno, y son los siguientes: EDM01 (establecer y mantener el marco de gobierno de TI), EDM02 (asegurar la entrega de beneficios), EDM03 (asegurar la optimización de riesgos), EDM04 (Asegurar la optimización de recursos) y EDM05 (asegurar la transparencia). Estos procesos son descritos con detalle en el documento ISACA (2012c).

A continuación se describe la estructura y contenidos de la información de los procesos, tal como aparece en el documento mencionado: 
- $\quad$ Etiqueta de proceso: El prefijo del dominio (EDM, APO, BAI, DSS, MEA) y el número de proceso.

- Nombre del proceso: Breve descripción, indicando el asunto principal del proceso.

- Área del proceso: Gobierno o gestión.

- Nombre de dominio: Nombre de cada dominio.

- Descripción del proceso: Visión general de lo que hace el proceso y una visión a alto nivel de cómo el proceso lleva a cabo su propósito.

- Declaración del propósito del proceso: Una descripción completa del propósito general del proceso.

- Información de la cascada de metas: Referencia y descripción de las principales metas relacionadas a TI que son soportadas por el proceso y métricas para determinar el logro de estas metas.

- Metas y métricas del proceso: Conjunto de metas del proceso y número limitado de métricas de ejemplo.

En la figura 5 se muestra, a modo de ejemplo, la información del proceso EDM01. 
Figura 5. Proceso EDM01. Descripción, propósito y métricas

\begin{tabular}{|c|c|}
\hline 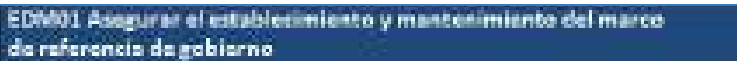 & 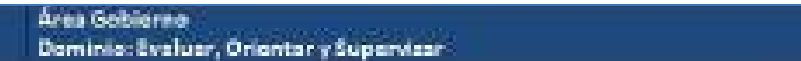 \\
\hline \multirow{2}{*}{\multicolumn{2}{|c|}{ 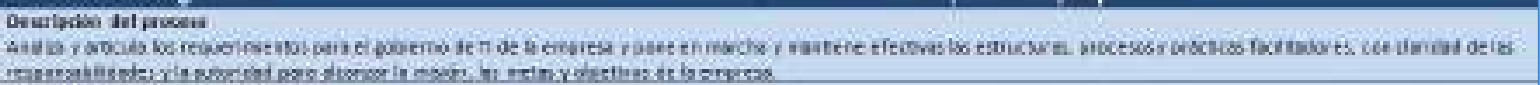 }} \\
\hline & \\
\hline \multirow{2}{*}{\multicolumn{2}{|c|}{ 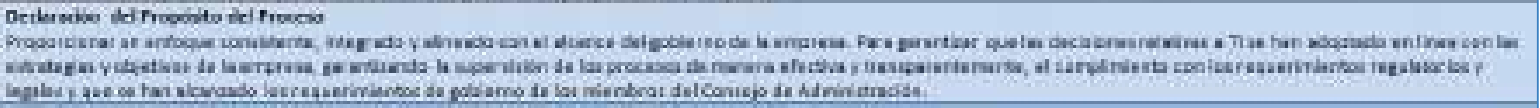 }} \\
\hline & \\
\hline \multicolumn{2}{|c|}{ 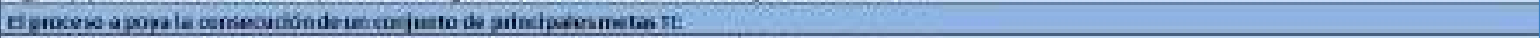 } \\
\hline Metan & Matricas mokiosantat \\
\hline \multirow[t]{3}{*}{ 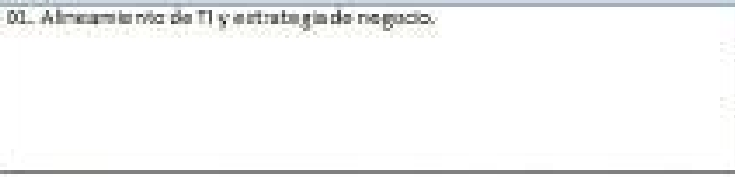 } & 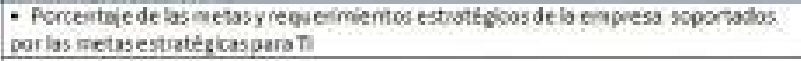 \\
\hline & 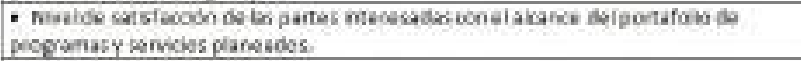 \\
\hline & 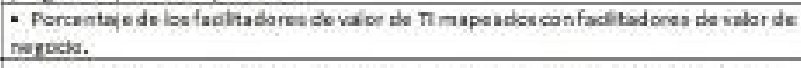 \\
\hline \multirow[t]{4}{*}{ 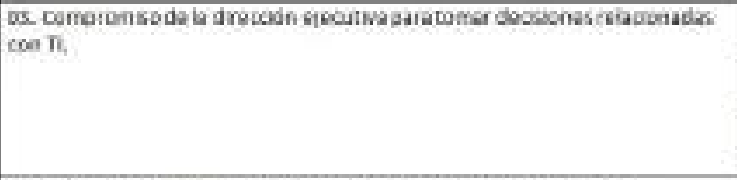 } & 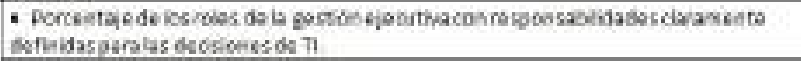 \\
\hline & 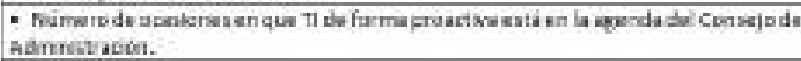 \\
\hline & 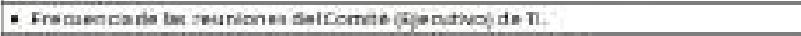 \\
\hline & 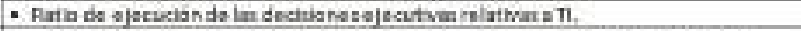 \\
\hline \multirow[t]{3}{*}{ 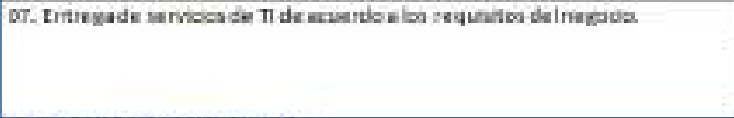 } & 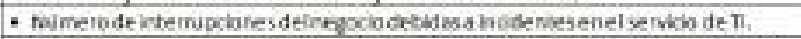 \\
\hline & 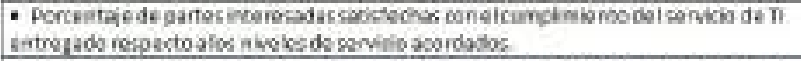 \\
\hline & 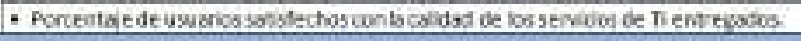 \\
\hline \multicolumn{2}{|l|}{ Mrtas y Metricat del Proseca. } \\
\hline 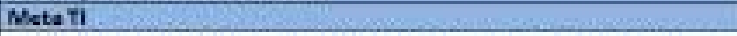 & Metrieas relosianades \\
\hline \multirow{2}{*}{ 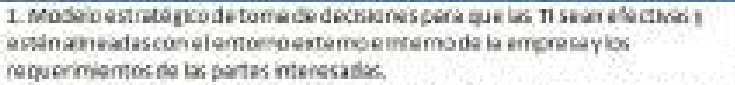 } & 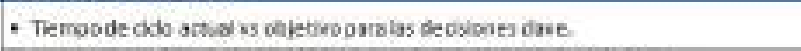 \\
\hline & 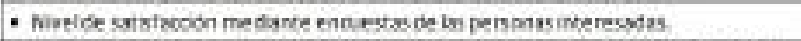 \\
\hline \multirow[t]{3}{*}{ 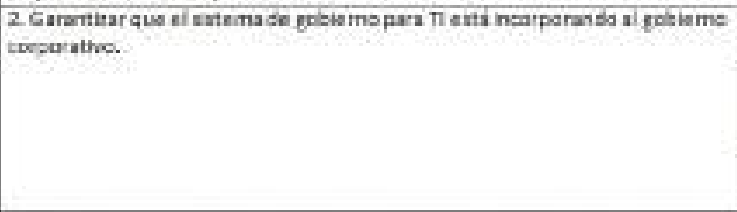 } & 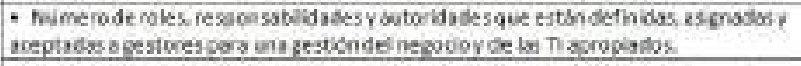 \\
\hline & 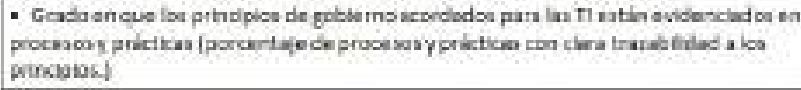 \\
\hline & 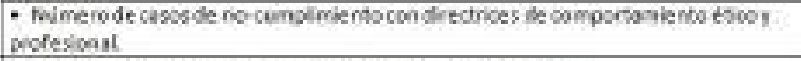 \\
\hline \multirow{3}{*}{ 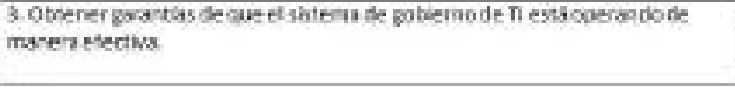 } & 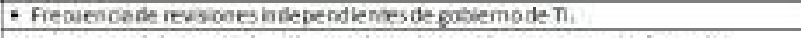 \\
\hline & 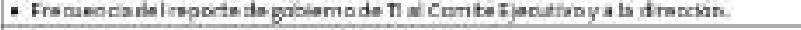 \\
\hline & 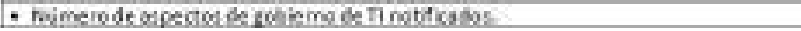 \\
\hline
\end{tabular}

Fuente: ISACA (2012c). Procesos Catalizadores - COBIT 5

- Matriz RACl: Asignación sugerida del nivel de responsabilidad para las prácticas del proceso a diferentes roles y estructuras. Los roles de la empresa están más sombreados que los roles de las TI. Los distintos niveles de implicación son: "R" (responsable): ¿Quién está haciendo la tarea? Hace referencia a los roles que se encargan de la actividad principal para completar la actividad y producir la salida esperada. "A" (responsable de que se haga) [del inglés, accountable]: ¿Quién rinde cuentas sobre el éxito de la tarea? "C" (consultado): ¿Quién proporciona entradas? Estos roles que proporcionan entradas son clave. "I" (informado): ¿Quién recibe la información? Estos son los roles que son informados de los logros o entregables de las tareas. En la figura 6, se muestra, como modelo, la información del proceso EDM01. 
Figura 8. Proceso EDM01. Guías relacionadas

\begin{tabular}{|c|c|}
\hline Estinctar rebodnnabs & Helesencia detailarts \\
\hline \multicolumn{2}{|l|}{ 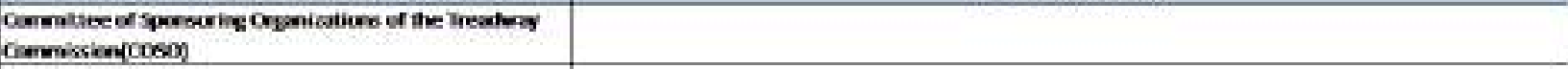 } \\
\hline \multicolumn{2}{|l|}{ Isa/ac:arm } \\
\hline & 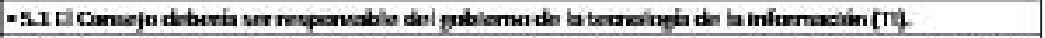 \\
\hline King il & 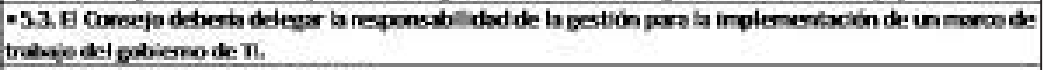 \\
\hline 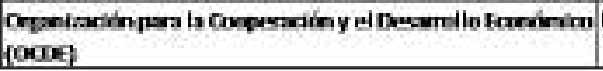 & Principies Corperatimes de tentiona \\
\hline
\end{tabular}

Fuente: ISACA (2012c). Procesos Catalizadores - COBIT 5

Nivel 2

En este nivel se identifican las actividades de mejora a ser implementadas en cada uno de los procesos de gobierno, a fin lograr el nivel de capacidad uno (proceso ejecutado) que garantice que los procesos están logrando sus metas. Para lograr este objetivo se utilizará el modelo de evaluación de procesos de COBIT 5 que está basado en la norma ISO/IEC 15504. En la figura 9 se aprecia un esquema del modelo de evaluación.

Figura 9. Modelo de capacidad de procesos

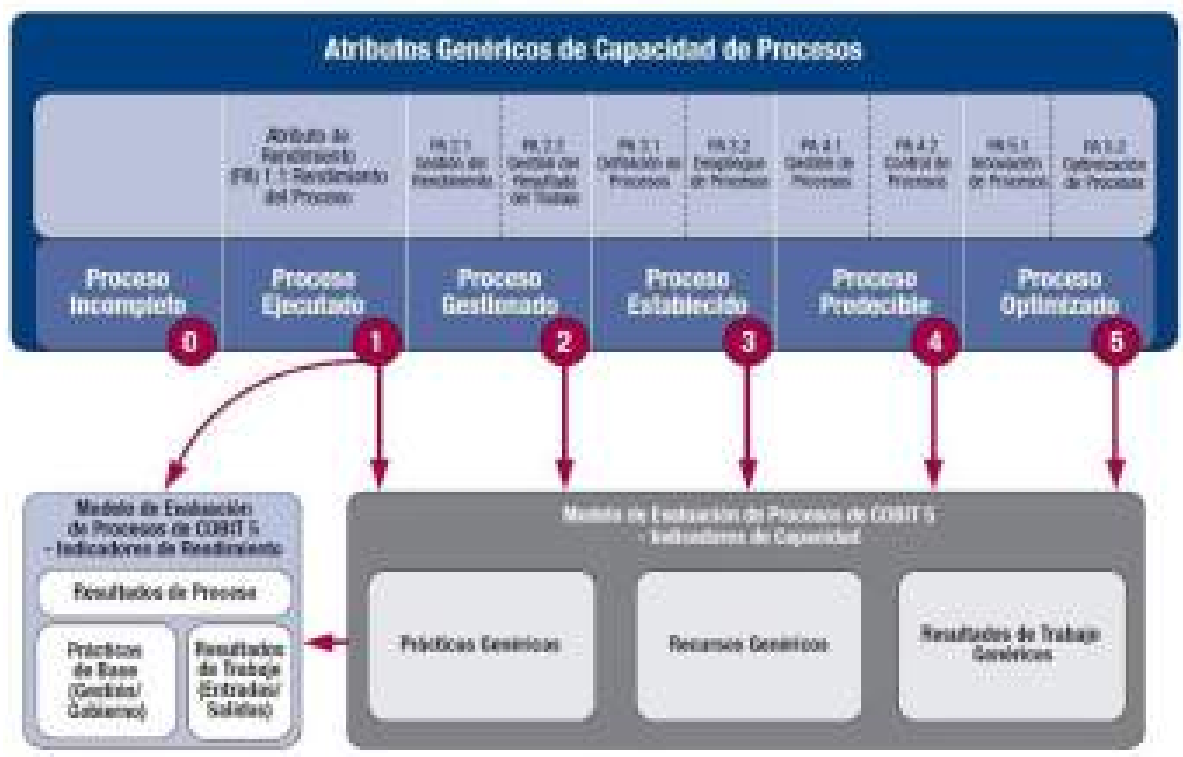

Fuente: ISACA (2012b). Un marco de negocio para el gobierno y la gestión de la empresa. 
En este nivel se utilizará la información de las actividades de los procesos presentados en el manual de procesos habilitadores de COBIT 5. Esta información servirá para determinar tanto su capacidad actual como las actividades de mejora correspondientes a cada uno de los procesos de gobierno. En el acápite 7.1 se presenta una herramienta desarrollada para efectuar las actividades de este nivel.

\section{Nivel 3}

En este nivel se hará un seguimiento al proceso de mejoras, para determinar si los procesos están logrando el nivel uno de capacidad (proceso ejecutado). Para lograr este objetivo se utilizarán las métricas de los procesos bajo análisis y servirán para determinar si los procesos están consiguiendo sus objetivos. En este nivel se usarán las métricas relacionadas a las metas de los procesos del manual de procesos habilitadores de COBIT 5, tal como se puede observar en la figura 5. En la figura 10 se presenta el Modelo correspondiente.

\section{Figura 10. Modelo de gobierno de las TI. Etapa 1 (GTIUP-1)}

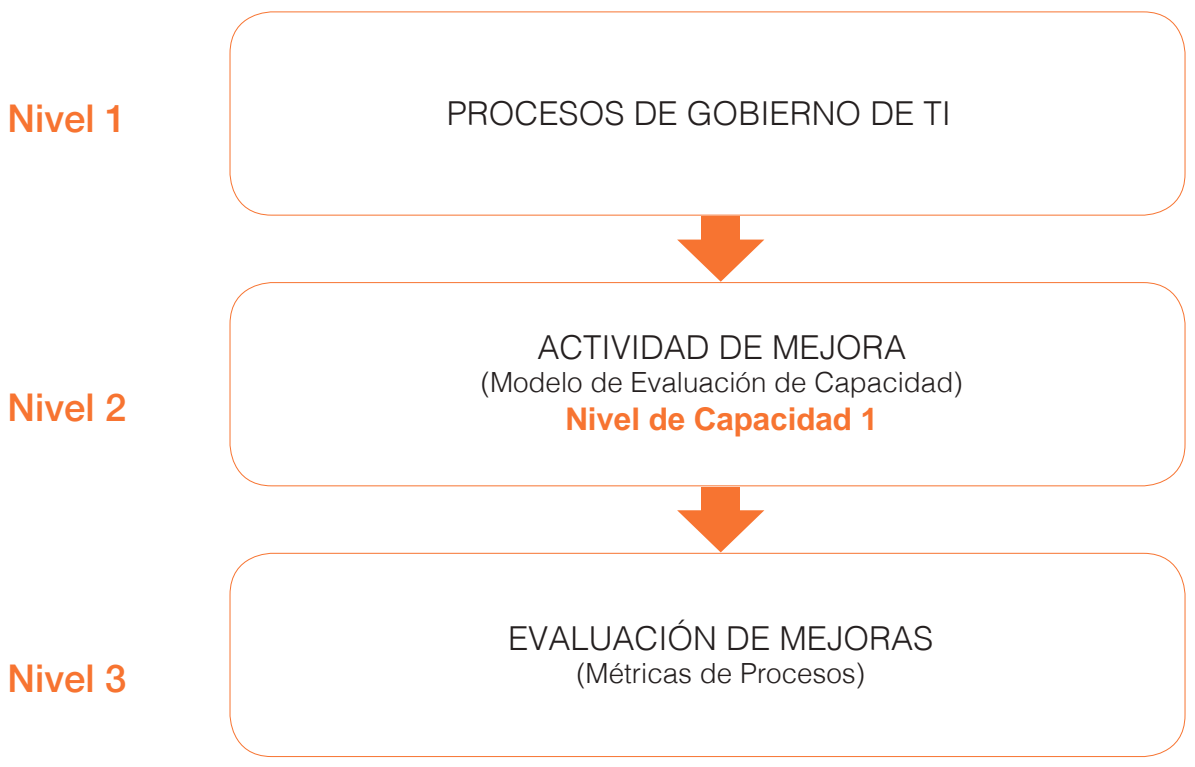

Elaboración propia

\subsection{Modelo de gobierno de las TI (GTIUP). Etapa 2}

Una vez que los procesos de gobierno de las TI hayan logrado el nivel 1 de capacidad (proceso ejecutado) siguiendo las pautas de la etapa 1 del modelo, se procederá a implantar la etapa 2 que permitirá identificar y mejorar los procesos de gestión de TI relevantes para la institución. Esta etapa está compuesta de los siguientes tres niveles: 
Nivel 1

En este nivel se deben identificar los procesos de gestión de las TI relevantes para la institución, los cuales deben ser analizados e implementados en el nivel de capacidad uno (proceso ejecutado). Estos procesos son los que darán el soporte a las políticas y directivas del nivel de gobierno para lograr el alineamiento entre la institución y las TI, posibilitando el desarrollo e implementación de soluciones de negocio que den soporte a los objetivos estratégicos de la organización. Para lograr este objetivo se utilizará la cascada de metas de COBIT 5. Este es un mecanismo que ayuda a traducir las necesidades de la institución en sus metas estratégicas, metas de las TI y procesos relevantes de TI. En la figura 11 se muestra un esquema de esta herramienta.

\section{Figura 11. Visión general de la cascada de metas de COBIT 5}

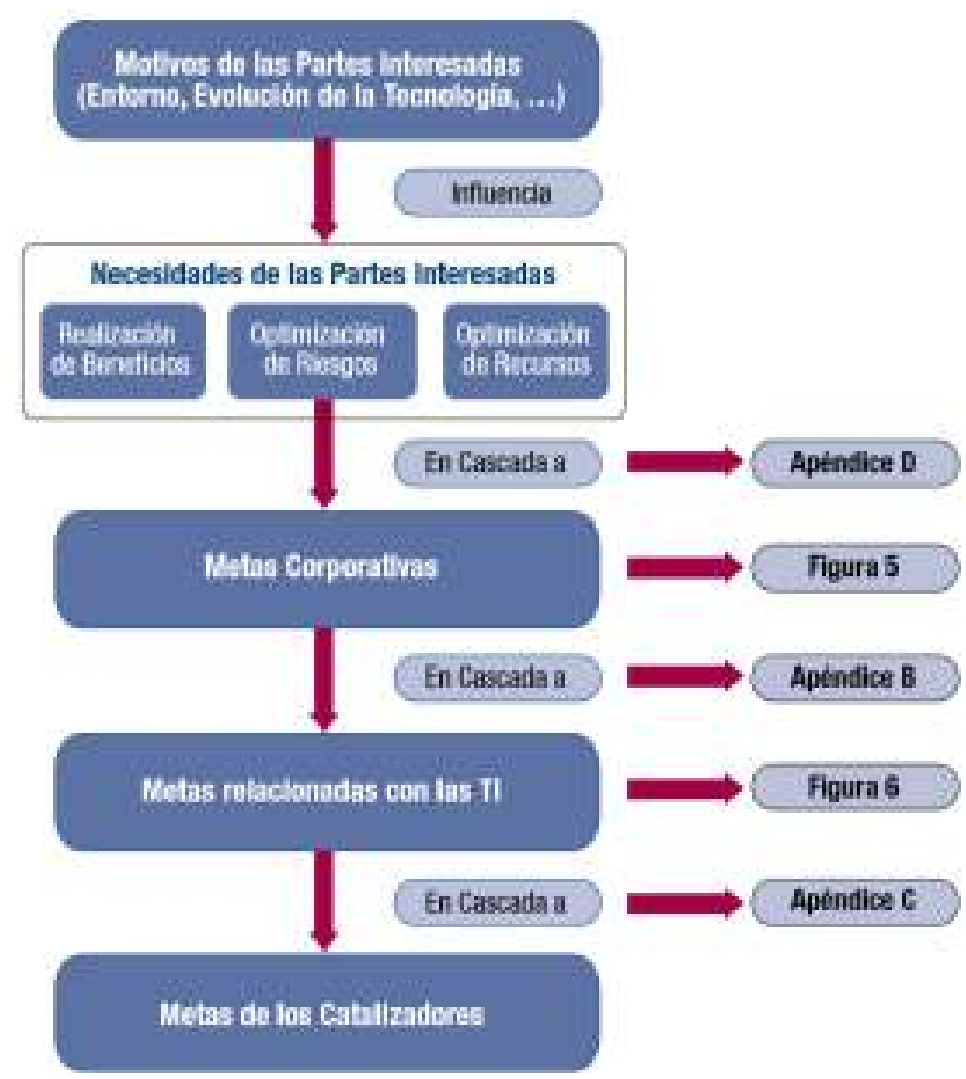

Fuente: ISACA (2012b). 
Con la ayuda de este mecanismo se podrán identificar los procesos de gestión relevantes para la institución. Como se mencionó anteriormente, esta tarea será efectuada en distintas fases considerando en cada fase no más de cinco procesos y durante un periodo no mayor a seis meses. En el acápite 7.2 se presenta una herramienta desarrollada para llevar a cabo las actividades de este nivel.

\section{Nivel 2}

En este nivel se identifican las actividades de mejora que deben ser implementadas en los procesos de gestión en cada una de las fases contempladas, a fin lograr el nivel de capacidad uno (proceso ejecutado) que garantice que los procesos están logrando sus metas. Para alcanzar este objetivo se utilizará el modelo de evaluación de procesos de COBIT 5, de la misma forma en la que se utiliza en el nivel 2 de la etapa 1 del modelo.

\section{Nivel 3}

En este nivel se hará un seguimiento al proceso de mejoras, para determinar si los procesos están logrando el nivel uno de capacidad (proceso ejecutado). Se sigue el mismo esquema definido para el nivel 3 de la etapa 1. En la figura 12 se presenta el modelo correspondiente:

Figura 12. Modelo de gobierno de las TI. Etapa 2 (GTIUP-2)

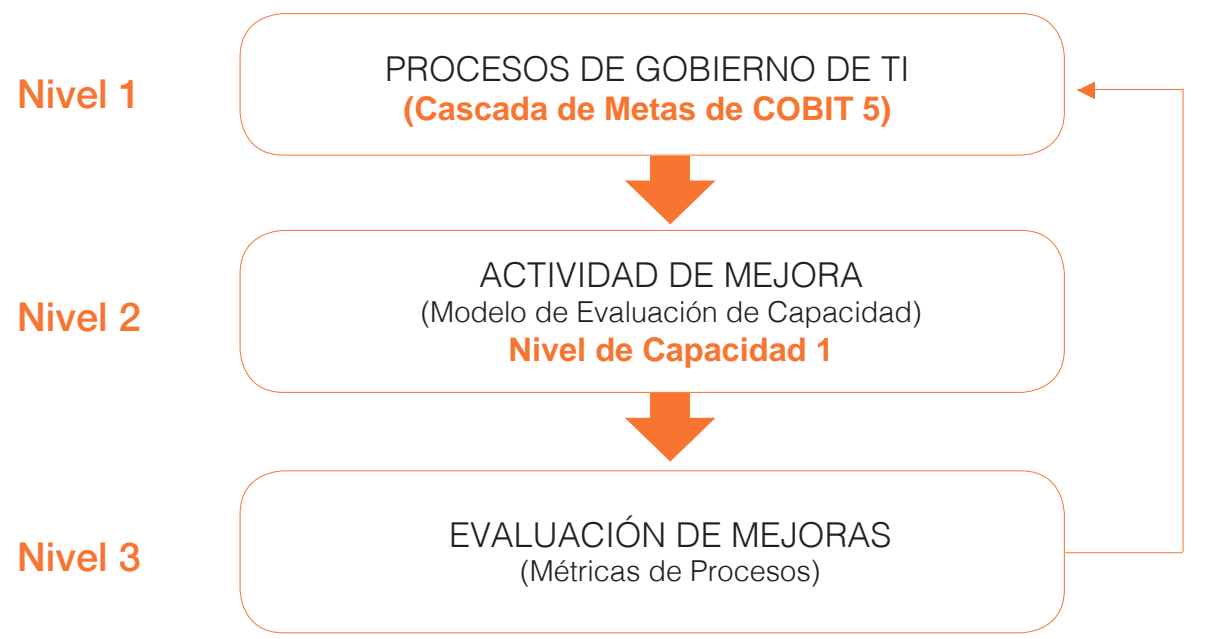

Elaboración propia

\subsection{Modelo de gobierno de las TI (GTIUP). Etapa 3}

La tercera etapa del modelo consistirá en la mejora continua del nivel de capacidad de los procesos de gobierno y gestión, teniendo como objetivo mejorar su nivel de capacidad por encima del nivel uno, en función a las necesidades de la institución. Al 
igual que en la segunda etapa, las actividades de mejora se llevarán a cabo en distintas fases. Esta etapa puede implementarse en forma paralela a la segunda etapa, o después de la etapa 2, en función a los objetivos de la institución.

\section{Consideraciones para la implementación}

Se procede a presentar y describir las herramientas desarrolladas en la presente investigación y que servirán de soporte en las fases iniciales del proceso de implementación del modelo propuesto. Estas herramientas han sido elaboradas en Excel y están basadas en la siguiente información de COBIT 5:

- Modelo de referencia e información detallada de los procesos (gobierno y gestión)

- Modelo de evaluación de capacidad de los procesos

- $\quad$ Cascada de metas

\subsection{Herramienta de evaluación de procesos}

Esta herramienta será de utilidad en la implementación de los niveles 1 y 2 de la etapa 1 del modelo propuesto y permitirá analizar los cinco procesos de gobierno e identificar las actividades de mejora para lograr el nivel 1 de capacidad (proceso ejecutado). Esta herramienta también será de utilidad al abordar el nivel 2 de la etapa 2 del modelo propuesto, permitiendo identificar las actividades de mejora de los procesos de gestión relevantes de la institución. La herramienta tiene información completa y estructurada de los treinta y siete procesos de COBIT 5 y para cada uno de estos procesos existen campos que permiten evaluar la capacidad actual del proceso a nivel de sus actividades con valores entre 0 y 100 \% que se anotan en el campo "Ingresar valores". Este valor significa el grado en el cual el proceso (actividad) en evaluación está alcanzando sus metas y tiene correlación con los estados N, P, L y F, conforme se determina en el modelo evaluación de COBIT 5, de acuerdo al siguiente esquema:

- "N": No se alcanzó (Not achieved). De 0 \% a 15 \%

- "P": Se alcanzó parcialmente (Partially). De 15 \% a 50 \%

- "L": Alcanzado en gran medida (Largely). De 50 \% a 85 \%

- "F": Totalmente alcanzado (Fully). De 85 \% a $100 \%$

Esta evaluación se debe realizar para cada una de las actividades de las prácticas de los procesos considerados. A modo de ejemplo, se presenta en la figura 13 la plantilla de 
la práctica EDM01.01 (evaluar el sistema de gobierno), del proceso EDM01 (asegurar el

establecimiento y mantenimiento del marco de referencia de gobierno).

Figura 13. Plantilla de evaluación de procesos. Inicial

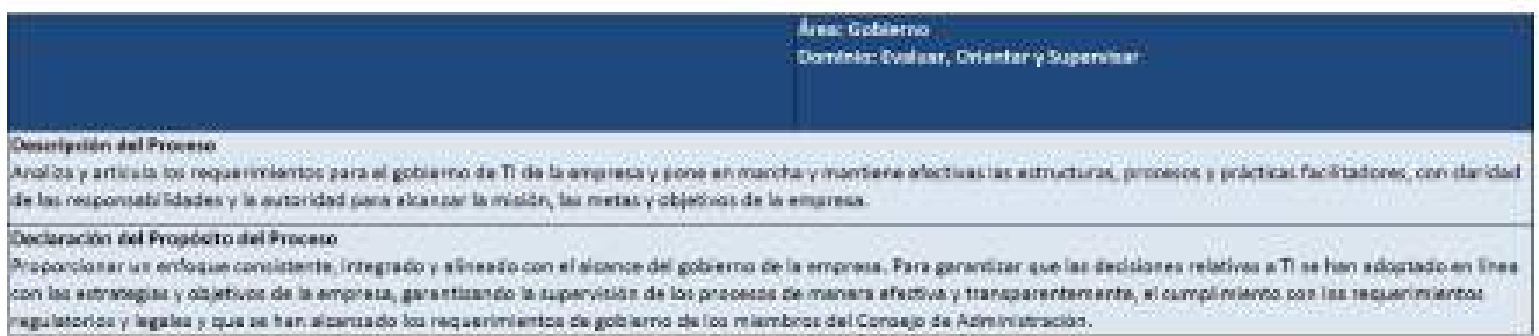

\begin{tabular}{|c|c|c|c|c|}
\hline \multirow{5}{*}{ 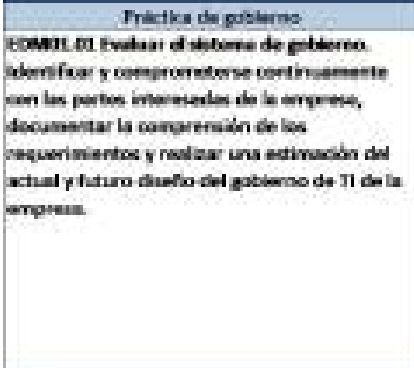 } & \multicolumn{2}{|r|}{ Entratas } & \multicolumn{2}{|c|}{ Salken } \\
\hline & De & Denctpotion & Destotpoth & A \\
\hline & Mesoa.02 & 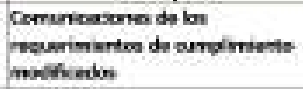 & Mrrapose chectrices del & 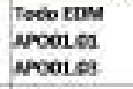 \\
\hline & \multirow[t]{2}{*}{$\begin{array}{l}\text { Fueradel } \\
\text { hinbta de } \\
\text { coart }\end{array}$} & \multirow{2}{*}{ 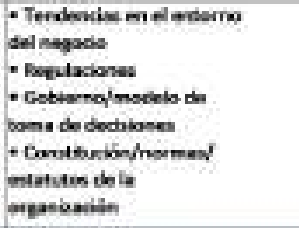 } & Medele de ieves da decidares & $\begin{array}{l}\text { Todo fDM } \\
\text { acolea. }\end{array}$ \\
\hline & & & \multirow[t]{2}{*}{ Mresien de musaridsed } & $\begin{array}{l}\text { Todo tra } \\
\text { Hower }\end{array}$ \\
\hline \multicolumn{4}{|c|}{ Mcthidader } & \\
\hline \multicolumn{5}{|c|}{ 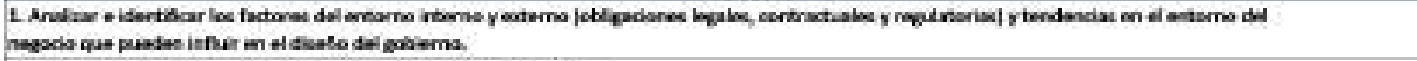 } \\
\hline \multicolumn{5}{|c|}{ 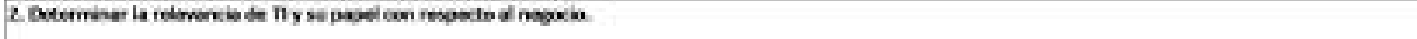 } \\
\hline \multicolumn{5}{|c|}{ 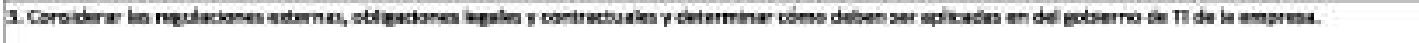 } \\
\hline \multicolumn{5}{|c|}{ 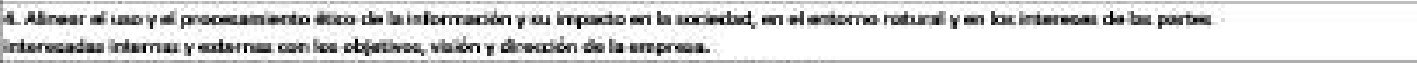 } \\
\hline \multicolumn{5}{|c|}{ 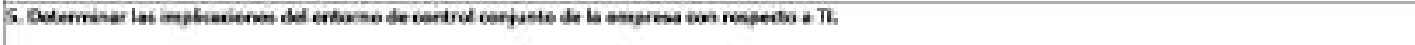 } \\
\hline \multicolumn{5}{|c|}{ 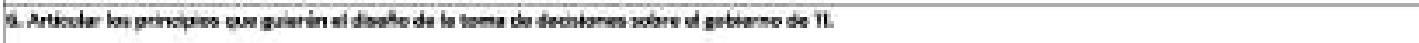 } \\
\hline \multicolumn{5}{|c|}{ 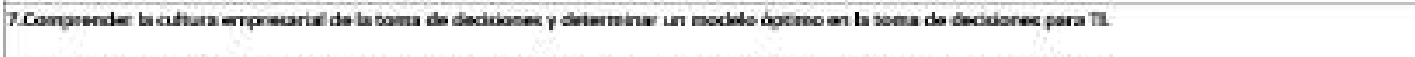 } \\
\hline \multicolumn{5}{|c|}{ 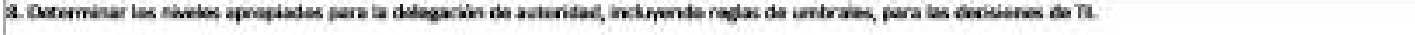 } \\
\hline
\end{tabular}



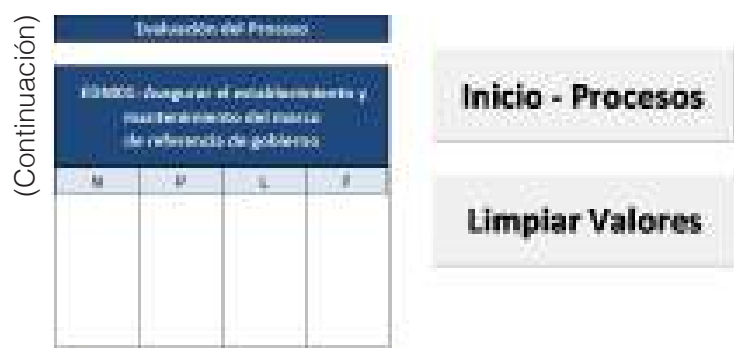

Limpiar Valores

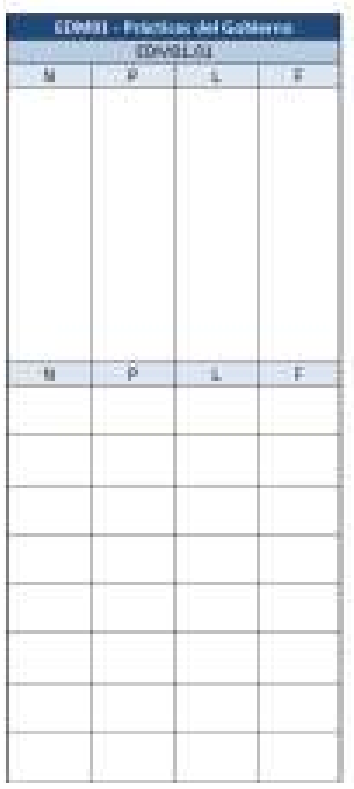

\section{Inicio - Procesos}

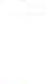

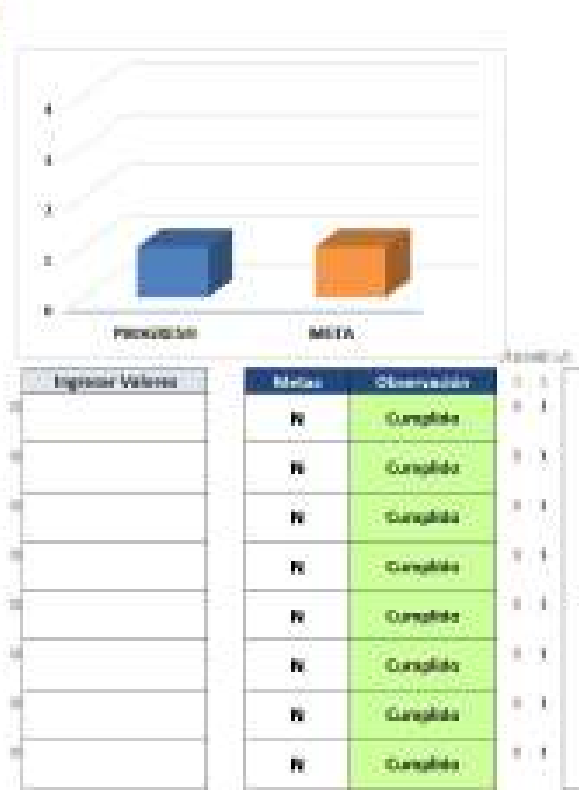

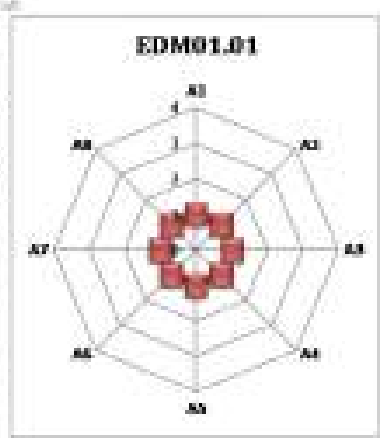

Elaboración propia

En la figura 14 se muestra la evaluación de capacidad efectuada a las actividades de la práctica EDM01.01. 
Figura 14. Plantilla de evaluación de procesos. Evaluación capacidad actual

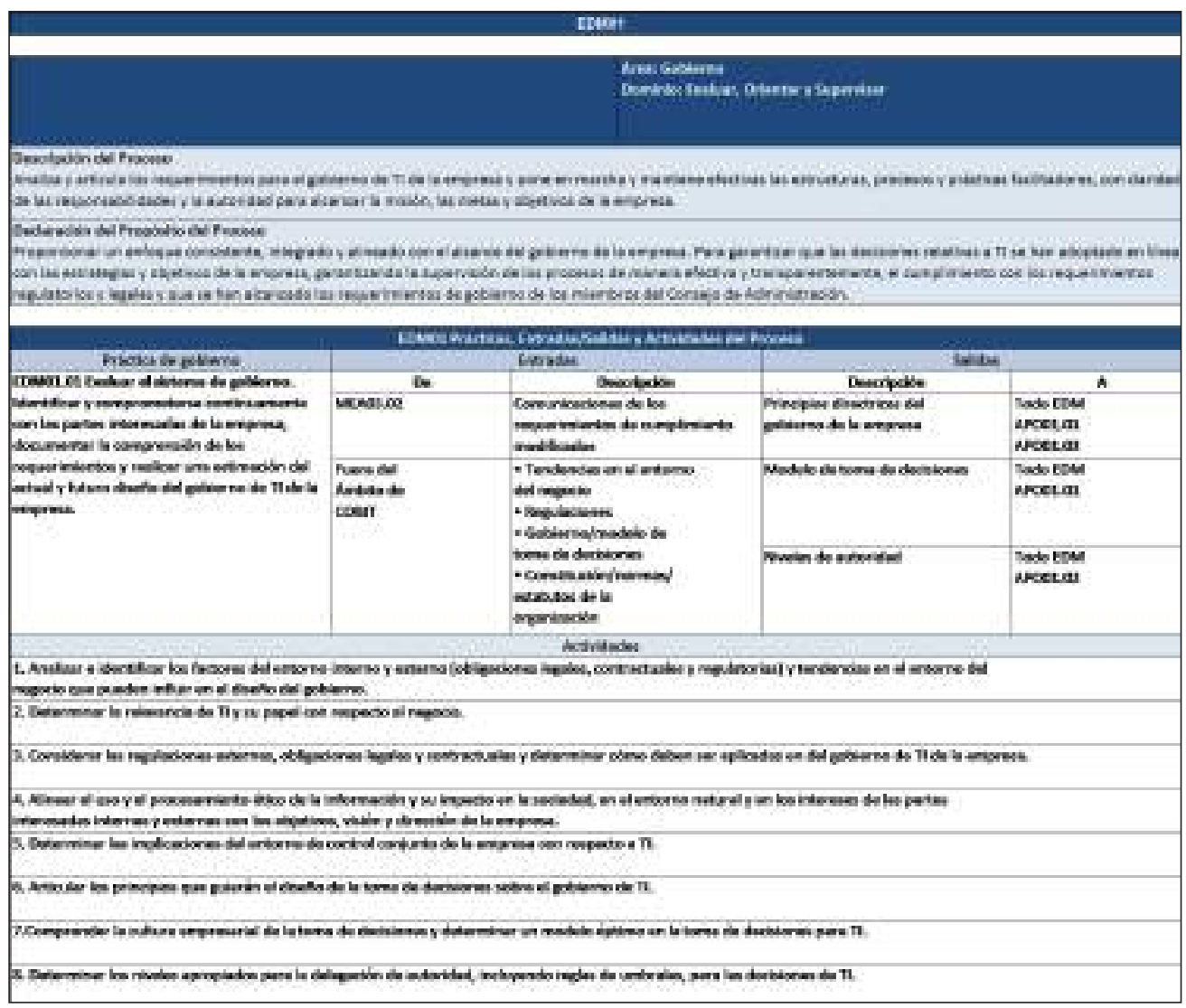

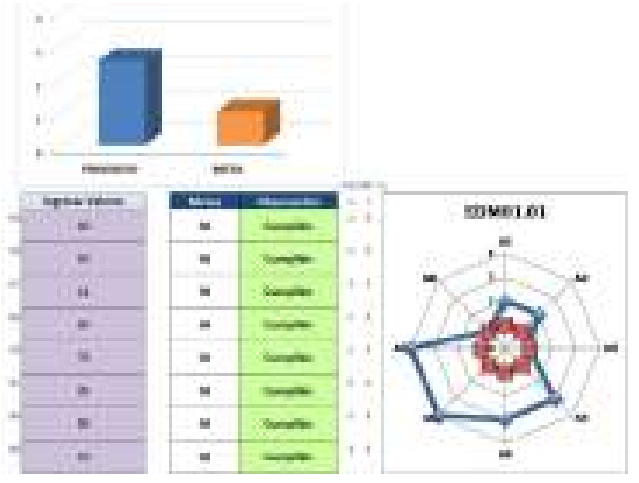

\begin{tabular}{|l|l|} 
Inicio - Procesos & Restaurar Metas \\
\hline Limpiar Valores & Restaurar Prioridades \\
\hline
\end{tabular}

Elaboración propia

Luego de evaluar la capacidad actual de las actividades, se debe definir el nivel de capacidad objetivo de la institución; en este caso la capacidad objetivo será "F" (totalmente alcanzado), a fin de lograr que la actividad, la práctica o el proceso en cuestión alcance el nivel 1 de capacidad (proceso ejecutado), conforme a lo establecido en el modelo propues- 
to. Esto se obtiene seleccionando en el campo "Metas" el estado "F". Al definir la meta de capacidad, la plantilla Excel marcará de forma automática el mensaje en rojo "No cumplido" en el campo de "Observación", si es que la capacidad actual de la actividad está por debajo de la meta de capacidad definida. Simultáneamente resaltará en color amarillo estas actividades, indicando que deben ser mejoradas (actividades de mejora) para llegar al nivel objetivo. En el caso de que el nivel de capacidad actual de la actividad fuese mayor o igual a la capacidad objetivo, el mensaje que se mostrará es el de "Cumplido". En la figura 15 se observan los resultados.

Figura 15. Plantilla de evaluación de procesos. Actividades de mejora

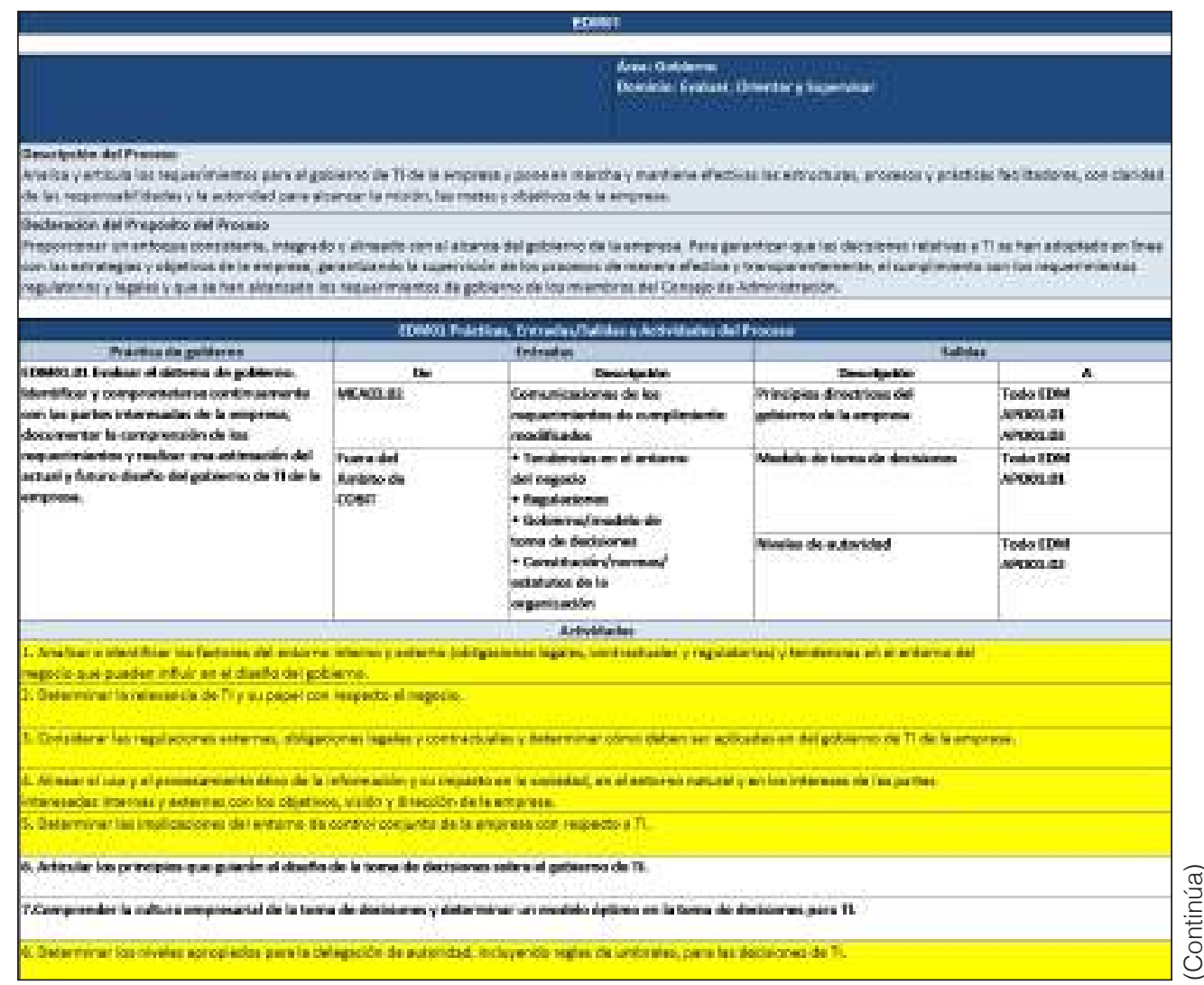




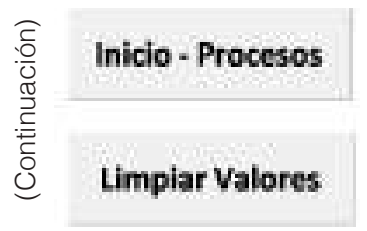

\section{Restaurar Metas}

\section{Restaurar Prioridades}

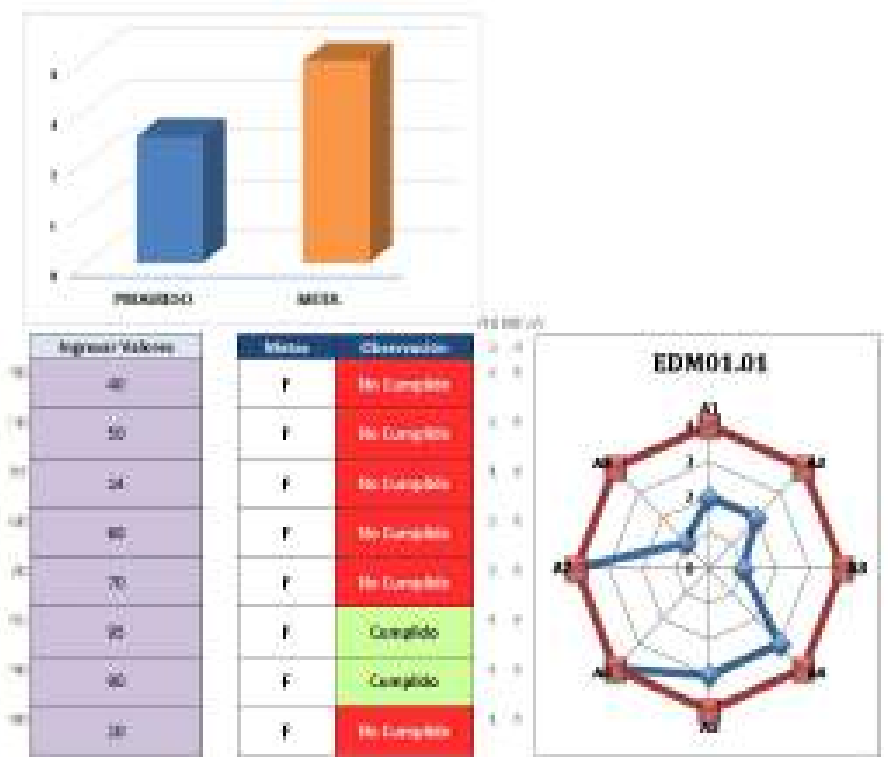

A fin de determinar la prioridad de ejecución de las actividades de mejora (resaltadas en color amarillo), se debe utilizar la columna de la derecha y dar valores a los parámetros:

- PE: Probabilidad de éxito de la actividad de mejora

- IPN: Impacto positivo en el negocio de las actividades de mejora

Esta priorización se efectúa tanto a nivel de actividad como a nivel de práctica para cada proceso evaluado, tal como se expone en la figura 16. 
Figura 16. Plantilla de evaluación de procesos. Priorización

\begin{tabular}{|c|c|c|c|c|}
\hline \multicolumn{5}{|c|}{ cast } \\
\hline \multicolumn{5}{|c|}{ 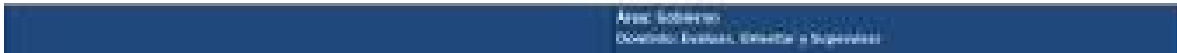 } \\
\hline \multicolumn{5}{|c|}{ 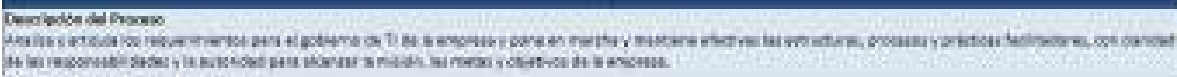 } \\
\hline \multicolumn{5}{|c|}{ 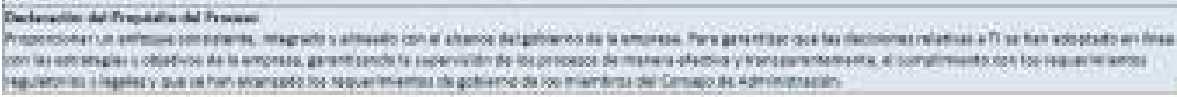 } \\
\hline \multicolumn{5}{|c|}{ 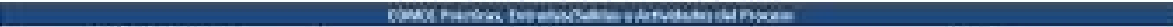 } \\
\hline \multirow{5}{*}{ 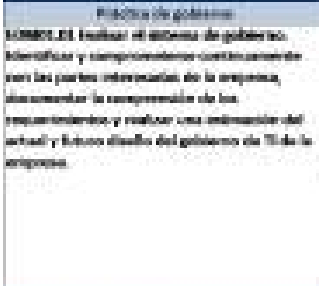 } & & Imiven & sent & \\
\hline & a & anches & sinew & 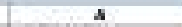 \\
\hline & vovanas & 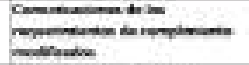 & 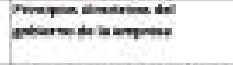 & 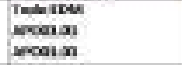 \\
\hline & $\lim _{\text {now }}$ & 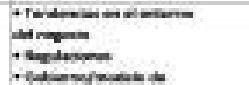 & 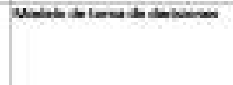 & 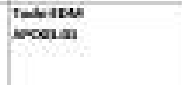 \\
\hline & & 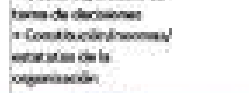 & Noliseritentet & 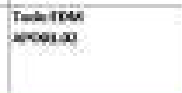 \\
\hline \multicolumn{5}{|c|}{ shatiden } \\
\hline \multicolumn{5}{|c|}{ 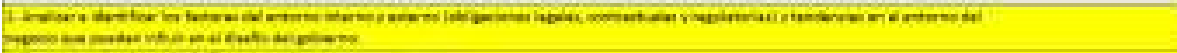 } \\
\hline \multicolumn{5}{|c|}{ 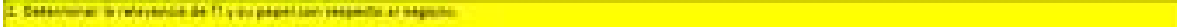 } \\
\hline \multicolumn{5}{|c|}{ 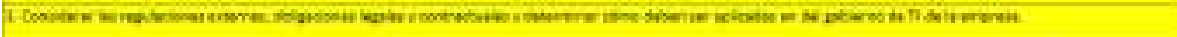 } \\
\hline \multicolumn{5}{|c|}{ 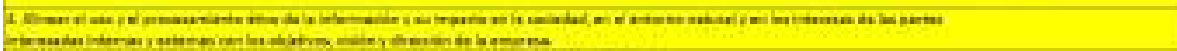 } \\
\hline \multicolumn{5}{|c|}{ 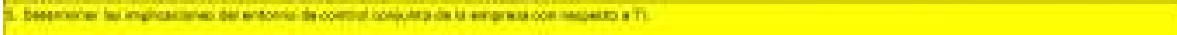 } \\
\hline \multicolumn{5}{|c|}{ 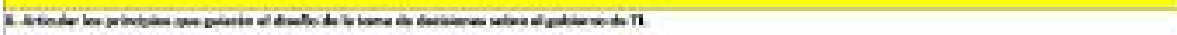 } \\
\hline \multicolumn{5}{|c|}{ 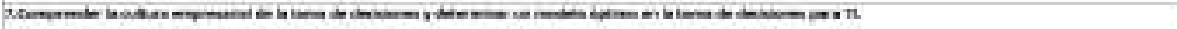 } \\
\hline 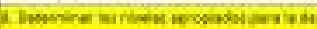 & wowesedines: & 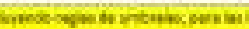 & kineserist & \\
\hline
\end{tabular}

\begin{tabular}{l} 
Iniclo-Proceses Restadrar Metas \\
Limpiar Valeres \\
\hline
\end{tabular}
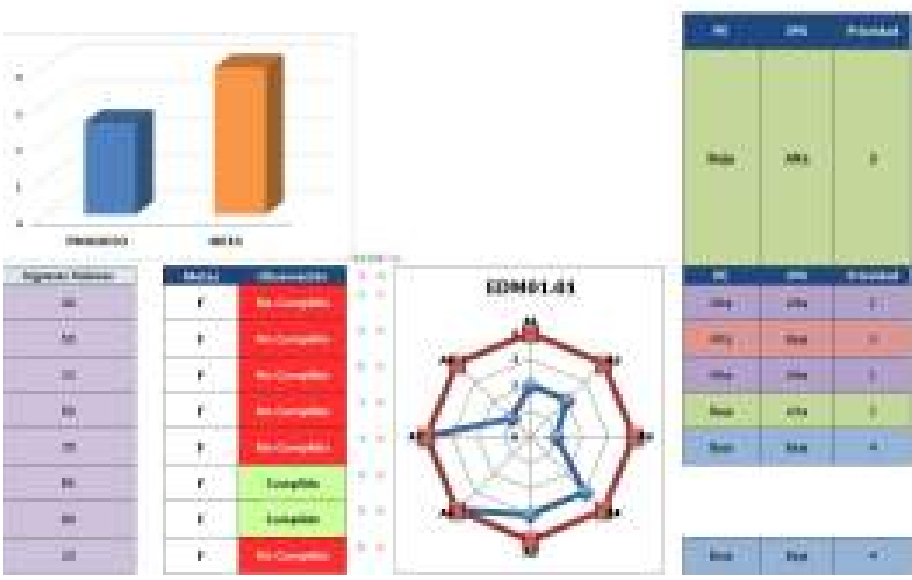

Elaboración propia 


\subsection{Herramienta de cascada de metas}

Esta herramienta será utilizada en la implementación del nivel 1 de la etapa 2 del modelo propuesto y determinará los procesos de gestión relevantes para la universidad. El proceso parte con la identificación de los objetivos estratégicos de la institución (metas relacionadas con la universidad); luego de ello se hace un mapeo (matriz de relación) con las metas relacionadas con el negocio. COBIT 5 propone diecisiete metas genéricas de negocio, agrupadas conforme las cuatro áreas del cuadro de mando integral. En este proceso se analizan detenidamente cada una de las metas de la universidad y se busca la relación existente con las metas del negocio. En la figura 17 se presenta parte de la plantilla, para un conjunto de metas tomadas como ejemplo.

Figura 17. Cascada de metas. Mapeo entre las metas de la universidad y del negocio.

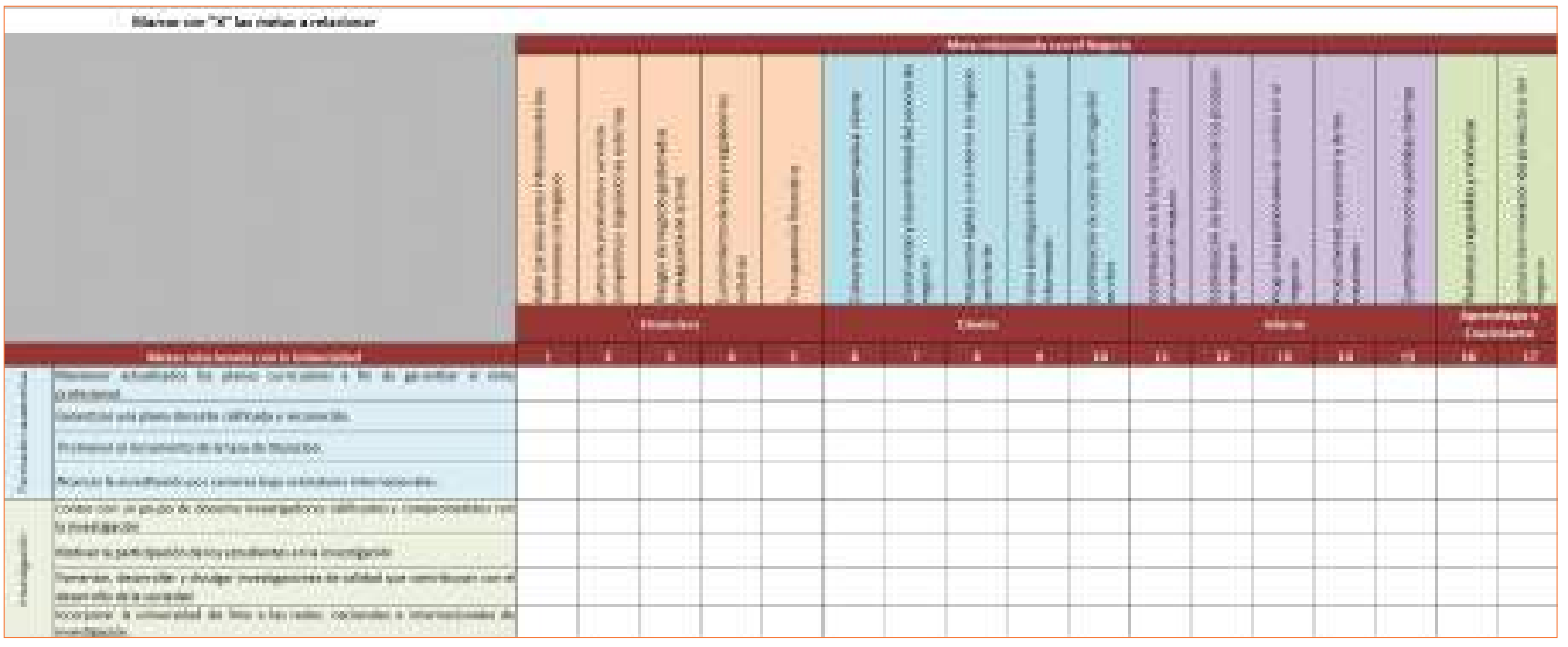

Elaboración propia

Una vez determinadas las metas de negocio, se hace un mapeo de las metas relacionadas con las TI. COBIT 5 propone diecisiete metas de TI genéricas, agrupadas según las cuatro áreas del cuadro de mando integral. En este proceso se determinarán las metas de TI relevantes para dar soporte a las metas de negocio, y por lo tanto, a las metas de la universidad. En la figura 18 se muestra la plantilla para lograr este objetivo. 
Figura 18. Cascada de metas. Mapeo entre las metas de negocio y las metas de TI.

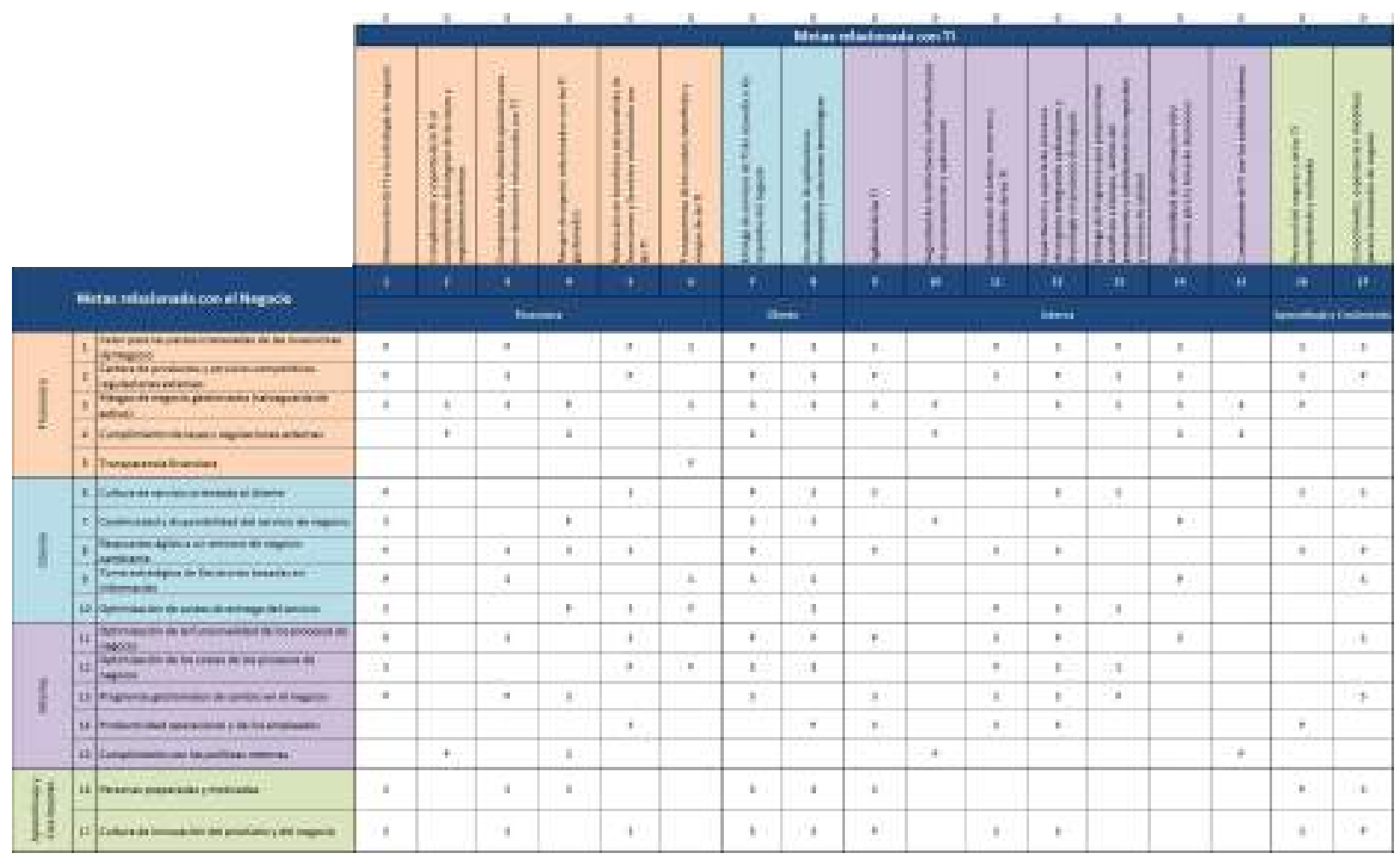

Elaboración propia

Con las metas de TI identificadas se efectuó un mapeo con los procesos de TI. COBIT 5 tiene treinta y siete procesos de TI agrupados en cinco dominios (un dominio de gobierno y cuatro de gestión). Como resultado se determinan los procesos de TI relevantes para la institución, que deberán ser evaluados para identificar las actividades de mejora correspondientes. En la figura 19 se observa la plantilla para lograr este objetivo. 
Figura 19. Cascada de metas. Mapeo entre las metas de TI y los procesos.

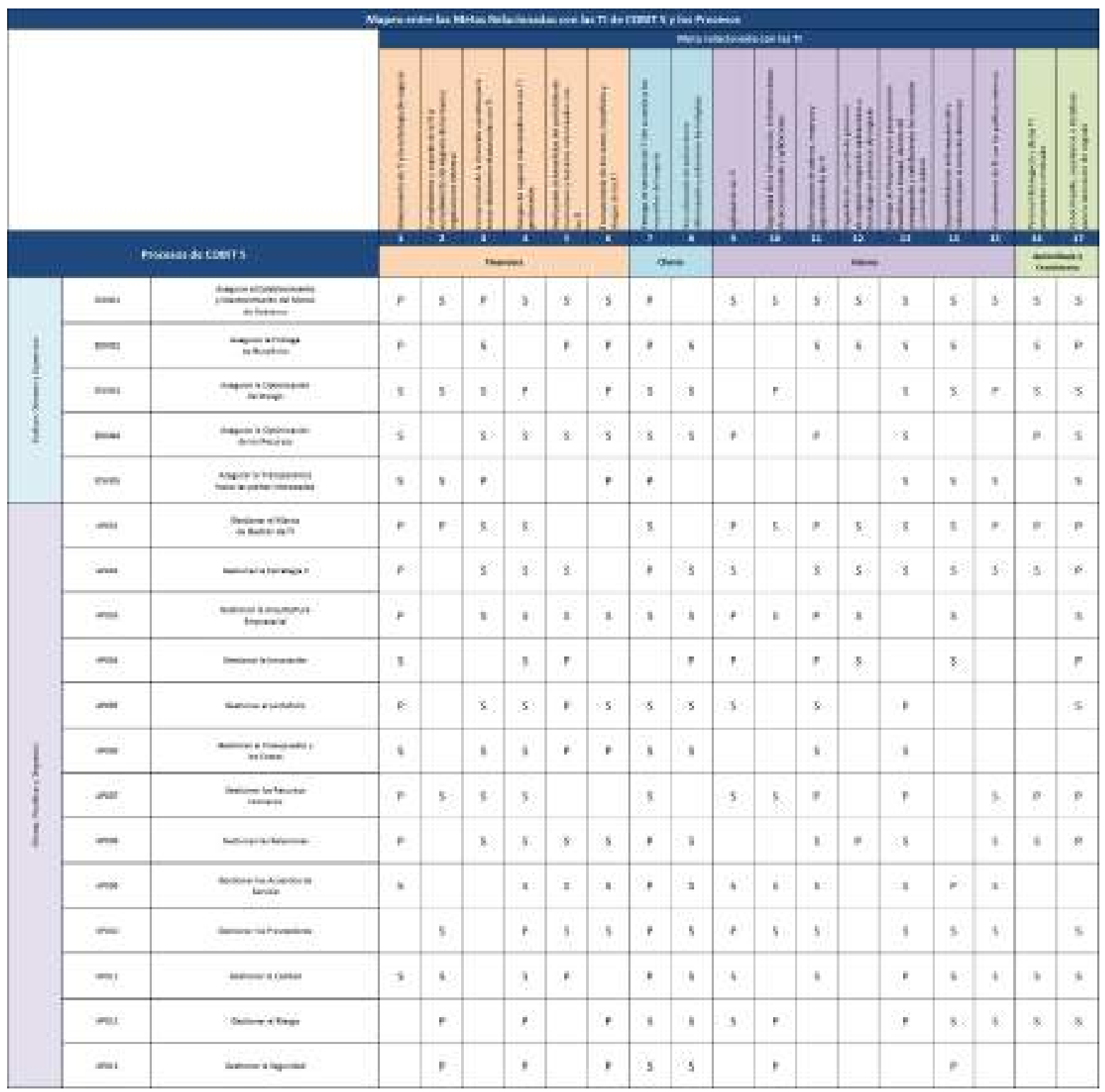

Elaboración propia

8. Conclusiones y recomendaciones

i. Las Tecnologías de la Información (TI) son un componente fundamental del sistema universitario, constituyéndose en una herramienta critica para el logro de los objetivos estratégicos de la institución. 
ii. El enfoque de gobierno de las tecnologías de la información, aplicado a las instituciones universitarias, propiciará el involucramiento de los niveles más altos de la institución, en la dirección y control de los sistemas y soluciones basados en TI, lo cual generará beneficios para la institución al optimizar los riesgos y el uso de los recursos.

iii. El marco de referencia más adecuado para implantar el enfoque de gobierno de TI en las instituciones universitarias es el COBIT 5.

iv. En la presente investigación se presenta un modelo de implantación en tres etapas, basado en COBIT 5, que ayudará a las instituciones universitarias a iniciar este importante proceso.

v. Es muy recomendable que personal de todos los niveles de las instituciones universitarias inicien al más breve plazo un proceso de capacitación sobre el enfoque de gobierno de las $\mathrm{TI}$ y sobre el marco de referencia COBIT 5.

\section{Referencias}

ISACA (2012a). COBIT 5 Implementación. Illinois: Autor.

ISACA (2012b). Un marco de negocio para el gobierno y la gestión de la empresa. Illinois: Autor.

ISACA (2012c). COBIT 5: Procesos catalizadores. Illinois: Autor.

ISACA (2014a). Certified in the Governance of Enterprise IT. Review Manual 2015. Illinois: Author.

ISACA (2014b). Global COBIT 5 Governance Study. Illinois: Autor. Recuperado de http://www. isaca. org/About-ISACA/Press-room/Documents/2014-Global-COBIT-Governance-StudyData-Sheet_pre_Eng_0914.pdf

ISACA (2015). Benchmarking and Business Value Assessment of COBIT 5. Illinois: Autor. Recuperado de http://www.isaca.org/knowledge-center/research/ researchdeliverables/ pages/benchmarking-and-business-value-assessment-of-cobit-5.aspx

International Organization for Standardization/International Electrotechnical Commission (ISO/ IEC) (2008). ISO/IEC 38500: Corporate Governance of Information Technology. Geneva, Switzerland: Autor

IT Governance Institute (2011). Global Status Report on the Governance of Enterprise IT. Rolling Meadows: Autor. Recuperado de http://www.isaca.org/knowledge-center/research/ researchdeliverables/pages/global-status-report-on-the-governance-of-enterprise-itgeit-2011.aspx 
